\title{
Is it necessary for screening irregular antibodies of blood donors?
}

\author{
Ziyi He*, Qingkai Chen, Shaobin Chen, Qing Wang, Jiaoli Zou, Zeming Liu, \\ Suyuan Huang, Zilang Zeng, Jialin Che \\ Dongguan Blood Center, Dongguan, Guangdong, 523930, China
}

\begin{abstract}
To explore the necessity of electronic crossmatching applied to irregular antibodies from blood donors, to ensure blood transfusion safety. Irregular antibody screening was performed on blood samples collected in the Dongguan Blood Center from Apr 17, 2014 to Dec 31, 2017. Primary screening was performed on the Sanquin automatic blood group analyzer by the microcolumn gel method (Sanquin). The positive samples were analyzed again using the salt medium method, polybrene method and micro-column gel method (Diana) for the second screening. If the second screening was positive, it was used to determine irregular antibody specificity (using panel cells) and any irregular antibody titer was detected. A total of 208,004 samples were detected, of which 316 were positive $(316 / 208,004 ; 0.152 \%)$. Among them, 120 alloantibodies $(120 / 135,139 ; 0.088 \%)$ were detected in male donors, which was much lower than in female donors $(173 / 72,865 ; 0.237 \%)(P<0.01)$. A total of 16 kinds of known irregular antibodies and 40 cases of unknown antibody specificity were detected, with 119 cases of IgG type and 197 cases of IgM type, at the ratio of 1.65:1. In female donors, the frequencies of anti-D, anti-E, anti-M and anti-Le ${ }^{\mathrm{a}}$ were significantly higher than in male donors $(P<0.01)$. In married couples, the frequencies of anti$\mathrm{D}$ and anti-E were significantly higher than those unmarried $(P<0.05)$. In minority nationalities, the frequency of anti-M was significantly higher than in the Han nationality $(P<0.05)$. In non-Guangdong donors, the frequencies of anti-D and anti-Le ${ }^{\mathrm{a}}$ were significantly higher than in Guangdong donors. 87.02\% of irregular antibody positive donors' antibody titers were lower than "++", which was deemed as no serious hazard for clinical transfusion. The study suggests that it is unnecessary to screen for irregular antibodies in blood donors. Male donors from Guangdong may not be required to undergo screening for irregular antibodies, and anti-D and anti-E only identification is also not required to be detected in female donors.
\end{abstract}

Keywords: donors, irregular antibodies, alloantibodies, autoantibodies, specificity

\section{INTRODUCTION}

Blood component transfusion can produce a series of immunological effects. Once the recipient is exposed to a non-ABO antigen, the immune system can develop irregular antibodies. Irregular antibodies

\footnotetext{
*Correspondence to: He Ziyi, MD, Department of Transfusion Research, Dongguan Blood Center, 19 Ningjing Road, Humen Town, Dongguan, Guangdong, 523006, China. TEL: 0086-769-85152673, E-mail: zyhe_8@163.com.

The authors declared no conflict of interests.
}

include alloantibodies and autoantibodies, with almost half of them being considered clinically significant; being related to hemolytic transfusion reactions or shortened red blood cell(RBC) survival. However, in mainland China, there are no regulatory requirements for all donors to be screened for irregular antibodies. Despite this, in developed countries such as those in Europe and the United States, it is listed as routine. If donors' blood components contain irregular antibodies, this may lead to a minor crossmatching compatibility, in which blood components are transfused to the recipient, causing possible hemolytic transfusion 
adverse-reactions. Another purpose of this study is to explore the basis for developing an electronic crossmatching program in China. Such an electronic crossmatch system has already been implemented in many developed counties, such as the United States, Denmark, Holland, Norway, Britain, Canada and Australia, in which irregular-antibody screening of all blood donors is necessary ${ }^{[1]}$. In order to explore the incidence and distribution of irregular antibodies in blood donors in China, and confirm the necessity of electronic crossmatching (as well as prevent wasteful spending and staff costs), we analyzed 208,004 cases of irregular antibody screening for blood donors.

\section{MATERIALS AND METHODS}

\section{Blood samples}

Samples were collected from Dongguan blood donors from Apr 17, 2014 to Dec 31, 2017, and were stored at $2-8{ }^{\circ} \mathrm{C}$ before screening. A total of 208,004 blood donors' samples were analyzed; of which 135,139 were male donors $(71.9 \%)$, while 72,865 were female $(39.1 \%)$. Among these, 9 minority nationalities from 19 provinces in China were represented. This study was approved by the Dongguan Blood Center Ethics Committee and all aspects of the study complied with the Declaration of Helsinki.

\section{Reagents and instruments}

Polybrene reagent, Antibody Screening cells (Shanghai Blood Biopharmaceutical Co., Ltd., China) and Screening Panels (123, Sanquin, Netherlands), Panel cells (Shanghai Blood Biopharmaceutical Co., Ltd., China). Microcolumn gel and Antiglobulin Cards (Sanquin, Netherlands), DG Gel Coombs(Diana, Grifols, Spain), Microcolumn gel Card Incubator (Diana Incubator, Grifols, Spain), Microcolumn gel Card Centrifuge (Dienofuge, Grifols, Spain), Centrifuge (KA-2200, KUBOTA, Japan), Automatic blood group
Analyzer (Sanquin, Netherlands).

\section{Screening and identification of irregular antibodies}

The First irregular antibody screening was completed by the Sanquin automatic blood group analyzer, with the column agglutination method and a 3-cell screen (Sanquin Panel 123). The positive samples were confirmed for antibody type by the salt medium method and the polybrene/microcolumn gel agglutination method (Diana) with antibody screening cells (Shanghai Blood Biopharmaceutical Co., Ltd). Irregular antibody specificity was identified by panel cells which showed a clear pattern of erythrocyte antigen coverage. If the antibodies agglutinated with all panel cells and the donor's erythrocyte, they were identified as an autoantibody. All samples with autoantibodies were identified for IgM type or IgG type at $4{ }^{\circ} \mathrm{C}$ and $37^{\circ} \mathrm{C}$ by the saline medium method and the polybrene method. If the agglutination reaction was enhanced at $4{ }^{\circ} \mathrm{C}$, and weakened at $37^{\circ} \mathrm{C}$, it was identified as a cold antibody.

\section{Statistical analysis}

SPSS13.0 was used for statistical analysis, and the $x^{2}$ test was used to determine the related irregular antibody's frequency to a significance level of $P<0.05$.

\section{RESULTS}

\section{Basic situation}

Irregular antibody frequency is reported at different rates in different regions (Table 1). The frequency in this study showed lower than in Japan, France, Latin America, Costa Rica, Brazil, or in Sichuan province in China, but higher than others. Antibody screening was done in all 208,004 blood donors from Apr 17, 2014 to Dec 31, 2017. Male to female antibody distribution, as well as the total number of donors is shown in Table 2, of which $100 \%$ were voluntary and all were healthy. Out of a total of 316 irregular antibody posi-

Table 1 Irregular antibody frequency was reported in different regions

\begin{tabular}{|c|c|c|c|c|}
\hline Authors & $N$ & Positive number & Positive rate $(\%)$ & Donors \\
\hline Plumbley JA et al. ${ }^{[2]}$ & & & 0.040 & U.S.A. military \\
\hline Tiwari AK et al. ${ }^{[3]}$ & 31,367 & & 0.009 & India \\
\hline Pahujaet S et al. ${ }^{[4]}$ & 7,768 & (1). 4 & 0.050 & New Delhi, India \\
\hline Kaur D et al. ${ }^{[5]}$ & 6,350 & 7 & 0.110 & India \\
\hline Ducos J et al. ${ }^{[6]}$ & 661,511 & 2,021 & 0.300 & France Paris \\
\hline Ducos J et al. ${ }^{[6]}$ & 269,162 & 2,138 & 0.790 & France Toulouse \\
\hline Garg N et al. ${ }^{[7]}$ & 47,450 & 46 & 0.090 & India New Delhi \\
\hline Garcia MA et al. ${ }^{[8]}$ & / & / & 0.190 & Latin America, Costa Rica \\
\hline Garcia MA et al. ${ }^{[8]}$ & I & I & 0.340 & Brazil \\
\hline Takeshita A et al. ${ }^{[9]}$ & 248,785 & 3,554 & 1.430 & Japan \\
\hline Li CY et al. ${ }^{[10]}$ & 36,287 & 571 & 1.574 & Sichuan, China \\
\hline Zhu JY et al. ${ }^{[11]}$ & 15,033 & 42 & 0.003 & Shaoguan, China \\
\hline This study & 208,004 & 316 & 0.157 & Dongguan, China \\
\hline
\end{tabular}


Table 2 Basic situation of total donors and antibody positive donors

\begin{tabular}{lcccccc}
\hline Donors & Total $/ n(\%)$ & Male $/ n(\%)$ & Female $/ n(\%)$ & M:F & $x^{2}$ & $P$ \\
\hline Total & $208,004(100)$ & $135,139(100)$ & $72,865(100)$ & $1.85: 1$ & $/$ & $/$ \\
Alloantibody positive & $293(0.141)$ & $120(0.088)$ & $173(0.237)$ & $0.69: 1$ & 74.10 & $<0.01$ \\
Autoantibody positive & $23(0.011)$ & $16(0.012)$ & $7(0.009)$ & $2.28: 1$ & 0.21 & $>0.05$ \\
\hline
\end{tabular}

tive cases $(316 / 208,004 ; 0.152 \%)$, specific alloantibodies were found in 293 samples, at a ratio of $0.141 \%$ to the total amount $(293 / 208,004)$, and autoantibodies were found in 23 samples, at the positivity rate of $0.011 \%(23 / 208,004)$. The specific alloantibodies ratio in the Lewis system was the highest, reaching $37.34 \%$ (118/316) with a positive ratio of $0.056 \%$; 74 cases in the Rh system, accounted for $23.418 \%$ (74/316) with a positive ratio of $0.031 \%$; 42 cases in MNSs system accounted for $13.29 \%$ (42/316) with a positive ratio of $0.020 \%$. The male to female ratio of total blood donors was found to be 1.85:1. The male to female ratio of alloantibody positive and autoantibody positive results were $0.69: 1$ and 2.28:1, respectively. The frequency of alloantibody positive results in females was higher than in the male participants $(P<0.01)$, but no significance was found between the males and females in the autoantibody positive results. There were 197 cases of IgM type irregular antibodies (197/316; 62.342\%), and 119 cases of IgG type irregular antibodies $(119 / 316 ; 37.658 \%)$. The ratio between them was 1.65:1.

The relationship of alloantibody distribution frequency between genders and marital status
The frequency of irregular antibody in Rh, MNSs and Lewis blood system were higher in females than in males $(P<0.01$, Table 3). This was also shown the frequencies for anti-D and anti-E in the Rh blood system, anti-M in MNSs blood system, and anti$\mathrm{Le}^{\mathrm{a}}$ in the Lewis blood system between genders were found statistically significant $(P<0.01)$. The results showed that the three blood system alloantibodies (MNSs, Lewis, P blood system) (170/293, 58.02\%) were the most frequently found in blood donors corresponding to non-clinically significant alloantibodies. In the other 123 cases $(123 / 293 ; 41.98 \%)$ clinically significant irregular antibodies were all found, $60.16 \%$ of them belonged to the Rh blood system, while $81.08 \%$ of irregular antibodies relating to the $\mathrm{Rh}$ blood system were found in female donors.

\section{The relationship of alloantibody distribution frequency between nationality and native place}

The frequency of anti-D in the Rh blood system was higher in donors outside Guangdong when compared to donors in Guangdong $(P<0.05)$; anti-M in the MNSs blood system were higher in the Han nationality $(P<0.01)$; anti-Le $\mathrm{e}^{\mathrm{a}}$ in the Lewis blood system were higher in donors in Guangdong than in donors

Table 3 The relationship of alloantibody distribution frequency between gender and marital status

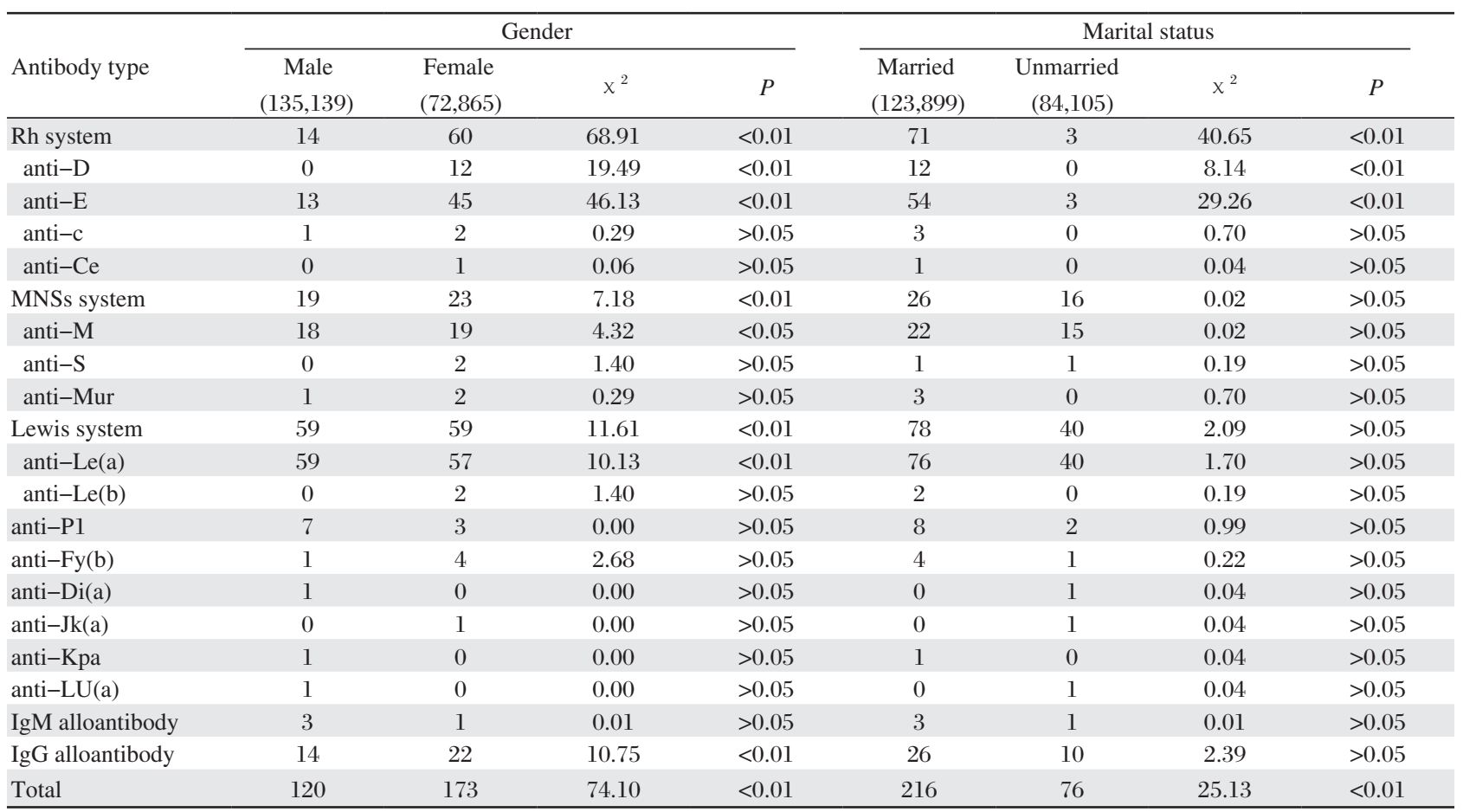


residing outside Guangdong $(P<0.01)$ (Table 4).

\section{The distribution of agglutination intensity}

The agglutination intensity of irregular antibody reaction is shown in Table 5. The agglutination intensity of "+" and below "+" was found in 201 cases $(65.12 \%)$, which was higher than the proportions of "++", "+++" and "++++" agglutination intensity $(P<0.01)$. The cumulative frequency below "++" reached to $87.02 \%$.

\section{DISCUSSION}

Irregular antibodies are produced by alloimmunization in cases of pregnancies or blood transfusions, and so on. They are called "irregular" due to target erythrocyte antigens being from "rare blood systems", and being separate from the ABO system. Irregular antibodies have been widely investigated in immunohematology since their presence in blood donors may

Table 4 The relationship of alloantibody distribution frequency between nationality and native place

\begin{tabular}{|c|c|c|c|c|c|c|c|c|}
\hline \multirow[b]{2}{*}{ Antibody type } & \multicolumn{4}{|c|}{ Nationality } & \multicolumn{4}{|c|}{ Native place } \\
\hline & $\begin{array}{c}\text { Han } \\
(197,272)\end{array}$ & $\begin{array}{l}\text { Minorities } \\
(10,732)\end{array}$ & $x^{2}$ & $P$ & $\begin{array}{c}\text { Guangdong } \\
(68,049)\end{array}$ & $\begin{array}{c}\text { Not Guangdong } \\
(139,955)\end{array}$ & $x^{2}$ & $P$ \\
\hline Rh system & 73 & 1 & 1.48 & $>0.05$ & 21 & 53 & 0.63 & $>0.05$ \\
\hline anti-D & 12 & 0 & 0.02 & $>0.05$ & 0 & 12 & 4.44 & $<0.05$ \\
\hline anti-E & 57 & 1 & 0.78 & $>0.05$ & 20 & 38 & 0.08 & $>0.05$ \\
\hline anti-c & 3 & 0 & 0.81 & $>0.05$ & 1 & 2 & 0.35 & $>0.05$ \\
\hline MNSs system & 36 & 6 & 5.40 & $<0.05$ & 17 & 25 & 1.73 & $>0.05$ \\
\hline anti-M & 31 & 6 & 7.11 & $<0.01$ & 14 & 23 & 0.44 & $>0.05$ \\
\hline anti-S & 2 & 0 & 1.60 & $>0.05$ & 2 & 0 & 1.62 & $>0.05$ \\
\hline anti-Mur & 3 & 0 & 0.80 & $>0.05$ & 1 & 2 & 0.35 & $>0.05$ \\
\hline Lewis system & 110 & 8 & 0.63 & $>0.05$ & 56 & 62 & 11.64 & $<0.01$ \\
\hline anti-Le(a) & 108 & 8 & 0.71 & $>0.05$ & 54 & 62 & 10.08 & $<0.01$ \\
\hline anti-Le(b) & 2 & 0 & 1.60 & $>0.05$ & 2 & 0 & 1.65 & $>0.05$ \\
\hline anti-P1 & 9 & 1 & 0.00 & $>0.05$ & 7 & 3 & 0.486 & $>0.05$ \\
\hline anti-Fy(b) & 4 & 1 & 0.24 & $>0.05$ & 1 & 4 & 0.017 & $>0.05$ \\
\hline anti-Di(a) & 1 & 0 & 1.00 & $>0.05$ & 0 & 1 & 0.13 & $>0.05$ \\
\hline anti-Jk(a) & 1 & 0 & 1.00 & $>0.05$ & 1 & 0 & 0.13 & $>0.05$ \\
\hline anti-Kpa & 1 & 0 & 1.00 & $>0.05$ & 1 & 0 & 0.13 & $>0.05$ \\
\hline anti-LU(a) & 1 & 0 & 1.00 & $>0.05$ & 0 & 1 & 0.13 & $>0.05$ \\
\hline IgM alloantibody & 3 & 1 & 0.44 & $>0.05$ & 2 & 2 & 0.04 & $>0.05$ \\
\hline IgG alloantibody & 36 & 0 & 1.04 & $>0.05$ & 16 & 20 & 2.25 & $>0.05$ \\
\hline Total & 275 & 18 & 0.57 & $>0.05$ & 256 & 38 & 16.17 & $<0.01$ \\
\hline
\end{tabular}

lead to difficulties in blood typing, blood crossmatching, or their side effects in inducing hemolytic transfusion reactions. According to the characteristics of antibodies, they can be divided into IgM type or IgG type. IgM type antibodies are cold-reactive antibodies (of which most are autoantibodies) and are generally known as non-clinically significant antibodies. IgG type antibodies are mostly clinically significant, and are mostly generated by immune stimulation, such as blood transfusions, pregnancy and/or organ transplantation. IgG type irregular antibodies can sensitize

Table 5 The intensity distribution of irregular antibody agglutination

\begin{tabular}{lccc}
\hline $\begin{array}{l}\text { Agglutination } \\
\text { intensity }\end{array}$ & $n$ & Frequency $(\%)$ & Cumulative frequency(\%) \\
\hline+ & 201 & 63.61 & 65.12 \\
++ & 74 & 23.41 & 87.02 \\
+++ & 26 & 8.23 & 95.25 \\
++++ & 15 & 4.75 & 100.00 \\
Total & 316 & 100.00 & \\
\hline
\end{tabular}

$\mathrm{x}^{2}=6.75, P<0.05$. erythrocytes, which may lead to delayed hemolytic transfusion reactions. In Europe, the United States and other developed countries, generally, donors are screened for irregular antibodies. Blood services in Hong Kong and Taiwan also screen for irregular antibodies as a routine. Plumbley et al. ${ }^{[2]}$ reported a positive rate of $0.04 \%$ for irregular antibodies from U.S. military donors, with only $0.02 \%$ testing positive for clinically significant irregular antibodies. Tiwari et $a l .{ }^{[3]}$ reported that the percentage of positive irregular antibodies from Indian donors was $0.09 \%$, which were mainly from anti-M in the MNSs system. Garg et $_{\text {al. }}{ }^{[4]}$ reported that a total of 46 donors showed the presence of alloantibodies $(46 / 47,450)$, yielding only a prevalence of $0.09 \%$. Most frequent alloantibodies identified were of the MNSs blood system. Pahuja et al. ${ }^{[5]}$ reported that 4 cases of alloantibodies were found in 7,768 blood donors from Teheran, with a positive rate of only $0.05 \%, 2$ of them were anti-C, 1 was anti-Le and 1 was autoantibody. The relative frequencies of irregular antibodies in donors were about $0.3 \%$ 
$(0.2 \%-0.8 \%)$ worldwide, $0.19 \%$ in Latin America and Costa Rica and $0.34 \%$ in Brazil ${ }^{[6]}$. In order to study the necessity irregular antibody screening on blood donors for development electronic crossmatching in China, we retrieved Chinese literatures published in recent years from CNKI, about 4,390,318 cases irregular antibody screen from 10 articles; where 545 cases being positive, at a total positive rate of $0.012 \%$, which was lower than that of France, New Delhi, USA, Latin America and Brazil. This was also significantly lower than the range of $0.2 \%-0.8 \%$ found in the frequency for donors worldwide. We speculated that perhaps the blood acquired from Chinese individuals is more homogenous than those from western countries, resulting in a lower frequency of irregular antibodies. However, Li et al. ${ }^{[7]}$ reported that in a total of 36,287 blood samples tested, 571 samples were irregular antibody positive, with a positive ratio of $1.574 \%(571 / 36,287)$, which is much higher than in previous Chinese literature. This may have something to do with different regions. In this report, from Apr 17, 2014 to Dec 31, 2017, all samples of blood donors were screened for irregular antibodies in the Dongguan Blood Center. A total of 208,004 cases were involved; 316(0.157\%) were positive, of which autoantibodies accounted for 23 cases, accounting for $0.011 \%$ of the total. Among all positive cases, 12 anti-D were all female, with 45 cases of anti-E were females, the frequencies were significantly higher than those in males $(P<0.01)$. $60.16 \%$ of all clinically significant alloantibodies belonged to the Rh blood system, of which $81.08 \%$ of the irregular Rh blood system antibodies were found in female donors. This is consistent with reports of McAdams et al ${ }^{[8]}$. In previous reports the irregular antibody frequency of Rh group system was the highest in female patients, and showed that the Rh group system (of alloantibody origin) is related to pregnancy ${ }^{[9,10]}$. In support of this, the frequency of irregular antibody in $\mathrm{Rh}$ blood system in married donors was also significantly higher than unmarried donors $(P<0.01)$, showing pregnancy as the reason for irregular antibodies in females. The frequencies of anti$\mathrm{M}$ and anti-Le $\mathrm{L}^{\mathrm{a}}$ in females were higher than those in males $(P<0.01)$. Because these were IgM type, males may be more exposed to variances in blood type material than females. The frequency of anti-M in the Han population was higher than in ethnic minorities $(P<0.01)$, which may be due to certain genetic and geographical factors.

The reagents and equipment of irregular antibody screening in this study were supplied by the Sanquin Company in the Netherlands. The Sanquin system has a high sensitivity. Due to limitations in panel cells, the identification of antibody specificity was also limited. Therefore, there were some uncertain antibody specificity (mostly IgG type) but their agglutination intensity was weak, with an in consistent reaction intensity when compared to the panel cells. According to the type of irregular antibody, 197 cases of $\operatorname{IgM}$ type irregular antibodies and 119 cases of IgG type irregular antibodies were detected, at the ratio of 1.65:1. A cumulative study of Chinese literature reported that 193 positive cases were detected in 572,959 donors, of which 182 were IgM type irregular antibodies, accounting for $94.3 \%$ of positive cases, and 11 were $\operatorname{IgG}$ irregular antibodies, accounting for $5.7 \%$ of positive cases, meaning that the positive rate of IgM type irregular antibodies was much higher than that of $\operatorname{IgG}$ type irregular antibodies. IgM type irregular antibodies are complete antibodies, which activate the complementary system - leading to acute hemolytic transfusion reactions. Chen et al. ${ }^{[1]]}$ detected 164 times of irregular antibodies in 121 cases of hemolytic transfusion reactions, of which 157 (95.73\%) were caused by IgG type irregular antibodies and 7 (4.27\%) were IgM type. Therefore, it is showing that in most cases irregular antibodies lead to hemolytic transfusion reactions.

According to the agglutination intensity, 15 cases were "++++", 26 cases were "+++", 74 cases were "++", and 201 cases were "+". In positive plasma, $65.12 \%$ of the irregular antibody agglutination intensity was weaker than "+" and $87.02 \%$ of the irregular antibody agglutination intensity was weaker than "++". Due to the extremely low frequency of irregular antibodies and most of them being non-clinically significance, some scholars believe it is not necessary to detect irregular antibodies for donors as a routine. China blood services supply frozen plasma and fresh frozen plasma for hospital, but acute hemolytic transfusion reactions caused by irregular antibodies transfused have not been reported. Acute hemolytic transfusion reactions usually occur in the $\mathrm{ABO}$ group system. And since IgG irregular antibodies generally cause delayed hemolytic transfusion reactions, they are easily ignored in clinic or masked by the original disease. Additionally, considering that RBC components (with irregular antibodies) would be diluted after transfusion, it is unlikely that they would cause the recipient a detectable problem. Even then, if the blood was transfused as fresh-frozen plasma, erythrocyte units, platelets, or cryoprecipitate, they would be diluted by the recipient's circulatory system, and unlikely to cause recipient hemolysis. Studies have shown that irregular antibodies targeting Duffy $\left(\mathrm{Fy}^{\mathrm{a}}\right)$, Kidd $\left(\mathrm{Jk}^{\mathrm{a}}\right)$ and $\mathrm{MNS}(\mathrm{M}, \mathrm{s})$ antigens in autoimmune 
patients suggest a loss of immune tolerance to these erythrocyte antigens ${ }^{[12]}$.

In summary, the rate of donors testing for irregular antibodies is very low, with most of these showing IgM type antibodies, which are generally considered to be non-clinically significant. IgG type irregular antibodies can cause RBC sensitization, however, as $87.02 \%$ of the antibody titers found in our study are below "++". So, even if the recipients contain the corresponding antigen for the antibody, the number of antigen-antibody reactions would be limited, and would not be enough to cause delayed hemolytic transfusion reactions. On clinic, the chances of hazardous hemolytic transfusion reactions caused by plasma testing positive for irregular antibodies from donors are very low. Therefore, from the perspective of clinical value and economic benefits, we do not believe it is a necessity for donors to be screened for irregular antibodies, or for female donors to be performed identification of anti-D and anti-E.

\section{References}

[1] He Z, Che J. Development and application of electronic crossmatch in Dongguan city. Asia-Pacific Journal of Blood Types and Genes, 2017, 1(4):37-41.

[2] Plumbley JA, Shelton JB Jr, Bowlus IN, et al. The incidence of unexpected antibodies in blood products collected at a military donor center. Mil Med, 1999, 164(10):705-6.

[3] Tiwari AK, Pandey P, Sharma J, et al. Incidence of clinically significant antibodies in patients and healthy blood donors: a prospective cross-sectional study from a tertiary healthcare center in India. TransfusApherSci, 2014,
50(2):230-4.

[4] Pahuja S, Kushwaha S, Sethi N, et al. Screening of blood donors for erythrocyte alloantibodies. Hematology, 2012, 17(5):302-5.

[5] Kaur D, Bains L, Kandwal M, et al. Erythrocyte alloimmunization and autoimmunization among blood donors and recipients visiting a Tertiary Care Hospital. J Clin Diagn Res, 2017, 11(3):EC12-5.

[6] Ducos J, Garretta M, Marty Y. Irregular antibody screening by Groupamatic at the Paris (C.N.T.S.) and Toulouse (C.R.T.S.) blood transfusion centers. Rev Fr Transfus Immunohematol(in French), 1978, 21(2):423-30.

[7] Li CY, Li YM, Huang F, et al. Characteristics of blood type irregular antibodies in Han population of Chinese Sichuan area. J Exp Hematol, 2015, 23(2):533-6.

[8] McAdams RM, Dantzler SA, Winter LW, et al. Severe hemolytic disease of the newborn from anti-e.g. Pernatol,2008, 28:230-2.

[9] Lin YS, Chang JS, Qiu Y, et al. Incidence of unexpected red blood cell antibodies in the north of China. Asia-Pacific Journal of Blood Types and Genes, 2017, 1(1):17-24.

[10] Qiao H, Xu J, Cai J, et al. Frequency of unexpected red blood cell antibodies in Nanjing. Asia-Pacific Journal of Blood Types and Genes, 2017, 1(2):67-70.

[11] Chen Z, Zhang L. Comprehensive analysis of hemolytic transfusion reaction and irregular antibody of non-ABO newborn hemolysis. Chinese Journal of Clinical Laboratory Science (in Chinese), 2001,19(6):377-8.

[12] López-Díaz PE, Ruiz-Olivera MDR, Hernández-Osorio LA, et al. Irregular antibodies in no hemolytic autoimmune diseases are able to induce erythrophagocytosis. Immunol Res, 2017, 65(1):410-8.

(Received 21 January 2018, Revised 08 February 2018, Accepted 03 March 2018) 